DOI: https://doi.org/10.46296/ig.v2i3.0006

\title{
OPTIMIZACIÓN DEL PROCESO DE ELABORACIÓN DE POUCH DE ATÚN EN “FISHCORP. S.A.”, ECUADOR
}

\author{
OPTIMIZATION OF THE PROCESS OF MAKING TUNA \\ POUCHES IN “FISHCORP. S.A.”, ECUADOR
}

\author{
Macías-López Diango ${ }^{1 *}$ \\ ${ }^{1}$ Universidad Internacional de la Rioja, UNIR. La Rioja, España.
}

*Correo: dmacias44@hotmail.es

\begin{abstract}
Resumen
En la actualidad es una realidad palpable la creciente popularidad de los envases flexibles tipo pouch, marcando una tendencia que destaca claramente en los procesos de producción alimenticia, y de manera especial en las conservas de atún, por su gran versatilidad, además de las numerosas ventajas frente a envases rígidos, como vidrio o latas. Entre estas ventajas destaca como principal factor un menor costo de producción y por ende márgenes de ganancia más holgados. Por esta razón se ha considerado realizar un proyecto en el cual se determine la factibilidad de optimizar el proceso de elaboración de pouch en la empresa Fishcorp. S.A, determinando diferentes aspectos que deberán ser considerados de resultar factible la implantación. Dentro del análisis técnico se determinan las restricciones que limitaban el crecimiento y fluidez del proceso, y mediante la aplicación de metodologías propias de ingeniería de procesos se identifica mejoras, que permitan el seguimiento de resultados con información real de producción y las variaciones positivas en términos de productividad y rentabilidad en el proceso.
\end{abstract}

Palabras clave: envases flexibles, pouch, Fishcorp. S.A, procesos, productividad, rentabilidad.

\begin{abstract}
Nowadays, the growing popularity of flexible pouch-type containers is a palpable reality, marking a trend that clearly stands out in food production processes, and especially in canned tuna, due to its great versatility, in addition to the numerous advantages vs. rigid packaging, like glass or cans. Among these advantages, the main factor is a lower cost of production and therefore more comfortable profit margins. For this reason, a project has been considered to determine the feasibility of optimizing the process of making pouch at the Fishcorp company. S.A., determining different aspects that must be considered if the implementation is feasible. Within the technical analysis, the restrictions that limited the growth and fluidity of the process are determined, and through the application of proprietary process engineering methodologies, improvements are identified that allow the monitoring of results with real production information and positive variations in terms of productivity and profitability in the process.
\end{abstract}

Keywords: flexible packaging, pouch, Fishcorp. S.A, processes, productivity, profitability.

Información del manuscrito:

Fecha de recepción: 05 de septiembre de 2018

Fecha de aceptación: 28 de noviembre de 2018

Fecha de publicación: 10 de enero de 2019 


\section{Introducción}

El presente proyecto tiene como finalidad establecer oportunidades de mejora en base a criterios técnicos, y determinar la factibilidad en términos económicos para su implantación. El potencial de crecimiento de una organización está determinado por la capacidad de la misma a adaptarse a los entornos cambiantes, tanto externos, como internos, y a la predisposición de la empresa por evolucionar de manera constante.

Este proyecto nace de la necesidad de la organización de mejorar de manera continua y sostenible, explotando sus procesos y aprovechando al máximo la capacidad instalada.

En el presente proyecto de carácter no experimental inductivo, se implementa un proceso productivo más eficiente que el actual en términos de costes y tiempo, además se identificaron los beneficios económicos para establecer la viabilidad.

\section{Metodología}

\subsection{Teoría de restricciones (TOC)}

La teoría de restricciones (TOC), es en esencia una filosofía de gestión empresarial, diseñada y presentada al medio industrial por el doctor Eliyahu Goldratt, esta filosofía gano gran popularidad a partir de su aparición estelar en su publicación "La meta", publicada por primera vez en 1984; este texto a modo de novela narra las problemáticas y oportunidades de mejora, presentes en un sistema formado por elementos interdependientes que al igual que en una cadena, sólo puede ser tan fuerte como su eslabón más débil, es decir, la restricción o cuello de botella (Eliyahu, 2005; Goldratt, 1994; Goldratt \& Cox, 2008).

La base de la teoría de las restricciones es ver a un sistema como un todo, siendo plenamente conscientes de que una empresa, una planta de producción, un almacén, una cadena de suministro, etc. es en realidad una serie de eslabones fuertemente dependientes entre sí. Aunque esto pueda parecer evidente, con frecuencia en la práctica se gestionan las diferentes partes del sistema como si fueran mucho más independientes. Pensemos, por ejemplo, en una empresa de cierto tamaño firmemente departamentalizada. Es bastante 
habitual que los diferentes departamentos (marketing, ventas, producción, atención al cliente, etc.) actúen persiguiendo objetivos locales. Así, cada uno de ellos intentará realizar optimizaciones en su área, esperando que la suma de las diferentes optimizaciones locales sea igual a la optimización global de la empresa. Desafortunadamente, esto no siempre genera buenos resultados. La empresa ha de verse como un todo y establecer una filosofía de mejora continua en la que, además de optimizar los procesos, se asegure la fluidez, la comunicación y la creación de valor entre ellos.

\subsection{Estudio de tiempos y movimientos}

Es innegable que dentro de las técnicas que se emplean en la medición del trabajo la más importante es el Estudio de Tiempos, o por lo menos es la que más nos permite confrontar la realidad de los sistemas productivos sujetos a medición.

El Estudio de Tiempos es una técnica de medición del trabajo empleada para registrar los tiempos y ritmos de trabajo correspondientes a los elementos de una tarea definida, efectuada en condiciones determinadas y para analizar los datos a fin de averiguar el tiempo requerido para efectuar la tarea según una norma de ejecución preestablecida (López, 2016).

\subsection{Diseño metodológico}

\subsubsection{Análisis técnico del proceso}

Se presenta un análisis detallado de los procesos concernientes a la línea de conservas de pouch, para lo cual se hará uso de la metodología teoría de restricciones (TOC), la cual consiste en iteraciones constantes de los pasos ya reconocidos, con el fin de eliminar progresivamente restricciones hasta llegar a un estado deseado o aceptable de fluidez dentro de la línea de proceso, junto con herramientas de análisis de tiempos para determinar las restricciones o cuellos de botella que limiten la capacidad del proceso.

Por tanto, llamaremos a cada una de estas iteraciones "ciclos", y en cada uno de estos se detallarán las condiciones coyunturales del mismo, hasta llegar al ciclo en el cual la totalidad de factores que determinan el avance del proceso y por tanto la fluidez de la línea estén balanceados. Es importante notar 
que estas iteraciones serán realizadas bajo las siguientes condiciones:

- Diferenciación para cada una de las presentaciones $(1 \mathrm{~kg}, 3 \mathrm{~kg}$ y $7 \mathrm{~kg})$.

- Las unidades estarán fijadas por la capacidad de planta.

- Por practicidad, en los casos en que se aumente la capacidad productiva, se priorizara por mantener las unidades $y$ disminuir los tiempos de proceso, y no mantener el tiempo aumentando las unidades a producir.

\section{Resultados y discusión}

\subsection{Primer ciclo TOC}

En el primer ciclo se presentan las condiciones del ciclo previo a la realización de cualquier tipo de cambio o modificación.

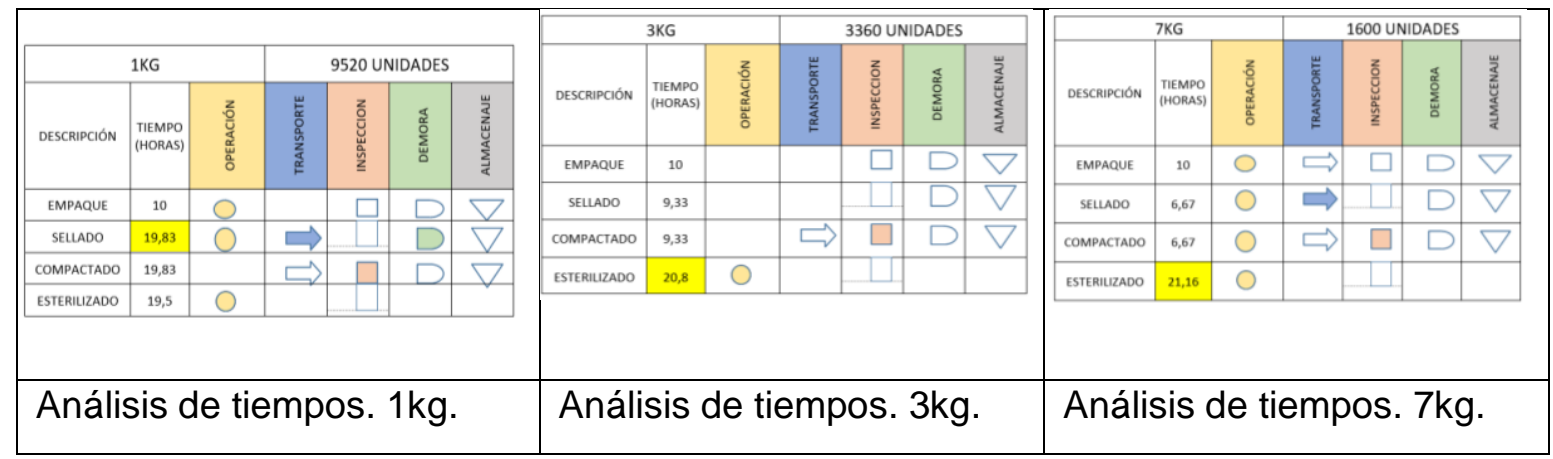

Figura 1. Primer ciclo TOC

Como se puede observar en la figura 1, correspondiente al primer ciclo para las presentaciones de $3 \mathrm{~kg}$ y 7 $\mathrm{kg}$ existe una clara restricción en el proceso de esterilización, representada como demora. Una vez identificada la operación que genera el retraso se procede a realizar el análisis respectivo, con el fin de levantar dicha restricción. Para este caso por tratarse de esterilización se presentan los tiempos relacionados con las autoclaves.

Tabla 1.

Tiempo de esterilización

\begin{tabular}{|l|l|l|l|l|}
\hline \multirow{2}{*}{ Formato } & Etapa & \multirow{2}{*}{ Tiempo total } \\
\cline { 2 - 4 } & Llenado y calentado & Esterilizado & Enfriamiento & \\
\hline 1 KG & $0: 18: 00$ & $1: 19: 00$ & $0: 40: 00$ & $2: 17: 00$ \\
\hline 3KG & $0: 18: 00$ & $1: 30: 00$ & $1: 10: 00$ & $2: 58: 00$ \\
\hline 7 KG & $0: 18: 00$ & $2: 25: 00$ & $1: 30: 00$ & $4: 13: 00$ \\
\hline
\end{tabular}

Fuente: Estudios de penetración de calor Fishcorp. S.A. (Ecuateinco. Cía.) 
Los tiempos para cada uno de los ciclos de esterilización mencionado anteriormente son obtenidos directamente de la receta generada para cada presentación en el estudio técnico de penetración y distribución de calor elaborado por la autoridad externa del proceso, por ende, se deben de manejar como tiempos estándar, es decir, no pueden sufrir alteraciones. De modo que, al trabajar con tiempos por parada predeterminados, la manera de establecer las cantidades máximas a producir está limitada por las paradas posibles en cada autoclave en un tiempo determinado.

En resumidas cuentas, la capacidad de las dos autoclaves disponibles esta al máximo y aun así no se cubren los tiempos requeridos por el proceso. Se plantea la adecuación de las condiciones de una tercera autoclave (\#3), misma que se encuentra deshabilitado y $\sin$ uso alguno hasta el momento. También se consideran complementos e insumos más representativos como coches y láminas de autoclavado adicionales.

Al incluir una autoclave más a las operaciones se pueden reducir los tiempos del proceso de Pouch o en su defecto mantener los tiempos actuales e incrementar la cantidad de producto esterilizado como se plantea en los siguientes escenarios: 1er Escenario. - Para este ejemplo en la tabla 2, se proyecta el uso del equipo esterilizando la misma cantidad de fundas, por ende, la disminución de tiempos con respecto a la utilización de solo 2 autoclaves.

- Formato $1 \mathrm{~kg}$ : 6:29 horas menos.

- Formato $3 \mathrm{~kg}$ : 6:58 horas menos.

- Formato $7 \mathrm{~kg}$ : 7:06 horas menos.

Tabla 2.

Reducción de tiempos de proceso

\begin{tabular}{|c|c|c|c|c|c|c|c|}
\hline \multirow{2}{*}{ Formato } & \multicolumn{2}{|c|}{ Autoclave 3 } & \multicolumn{2}{c|}{ Autoclave 4 } & \multicolumn{2}{c|}{ Autoclave 5 } & Total de \\
\cline { 2 - 7 } & $\begin{array}{c}\text { Unidades } \\
\text { esterilizada }\end{array}$ & $\begin{array}{c}\text { Tiempo } \\
\text { empleado }\end{array}$ & $\begin{array}{c}\text { Unidades } \\
\text { esterilizada }\end{array}$ & $\begin{array}{c}\text { Tiempo } \\
\text { empleado }\end{array}$ & $\begin{array}{c}\text { Unidades } \\
\text { esterilizada }\end{array}$ & $\begin{array}{c}\text { Tiempo } \\
\text { empleado } \\
\text { (Unid/Hora) }\end{array}$ \\
\hline 1 KG & 3173 & $12: 56$ & 3173 & $12: 56$ & 3173 & $12: 56$ & $9.520 / 13$ \\
\hline 3KG & 1120 & $13: 50$ & 1120 & $13: 50$ & 1120 & $13: 50$ & $3.360 / 14$ \\
\hline 7 KG & 533 & $14: 03$ & 533 & $14: 03$ & 533 & $14: 03$ & $1.600 / 14$ \\
\hline
\end{tabular}


2do Escenario. - En este ejemplo, se priorizan las unidades producidas, por tal motivo se generan más unidades de pouch manteniendo el tiempo establecido inicialmente.
- Formato $1 \mathrm{~kg}$ : 4775 Unidades más.

- Formato $3 \mathrm{~kg}: 1692$ Unidades más.

- Formato $7 \mathrm{~kg}: 809$ Unidades más.

\section{Tabla 3.}

Aumento de unidades esterilizadas

\begin{tabular}{|c|c|c|c|c|c|c|c|}
\hline \multirow[b]{2}{*}{ Formato } & \multicolumn{2}{|c|}{ Autoclave 3} & \multicolumn{2}{|c|}{ Autoclave 4} & \multicolumn{2}{|c|}{ Autoclave 5} & \multirow{2}{*}{$\begin{array}{l}\text { Total de } \\
\text { fundas } \\
\text { (Unid/Hora) }\end{array}$} \\
\hline & $\begin{array}{c}\text { Unidades } \\
\text { esterilizadas }\end{array}$ & $\begin{array}{l}\text { Tiempo } \\
\text { empleado }\end{array}$ & $\begin{array}{c}\text { Unidades } \\
\text { esterilizadas }\end{array}$ & $\begin{array}{l}\text { Tiempo } \\
\text { empleado }\end{array}$ & $\begin{array}{c}\text { Unidades } \\
\text { esterilizadas }\end{array}$ & $\begin{array}{l}\text { Tiempo } \\
\text { empleado }\end{array}$ & \\
\hline $1 \mathrm{KG}$ & 4765 & 19:25 & 4765 & $19: 25$ & 4765 & $19: 25$ & $14295 / 19$ \\
\hline $3 \mathrm{KG}$ & 1684 & $20: 49$ & 1684 & $20: 49$ & 1684 & $20: 49$ & $5052 / 20,5$ \\
\hline $7 \mathrm{KG}$ & 803 & $21: 09$ & 803 & 21:09 & 803 & $21: 09$ & $2409 / 21$ \\
\hline
\end{tabular}

\subsection{Segundo ciclo TOC}

Una vez realizadas las una nueva iteración con los análisis correspondientes.

modificaciones al proceso, se realiza

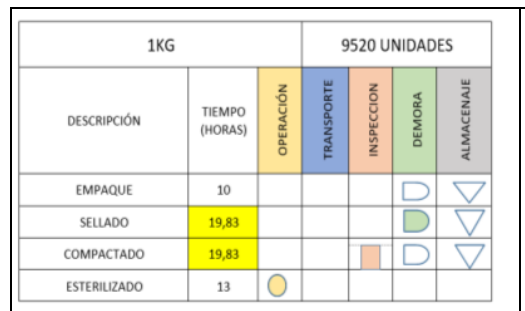

Análisis de tiempos. 1kg.

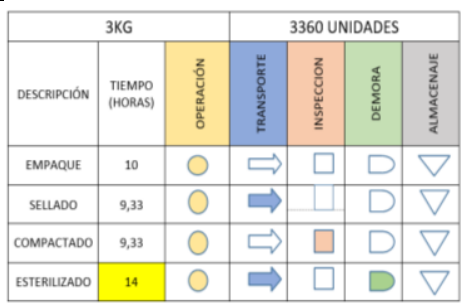

Análisis de tiempos. $3 \mathrm{~kg}$.

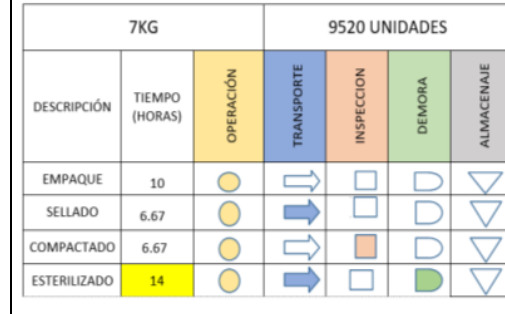

Análisis de tiempos. $7 \mathrm{~kg}$.

Figura 2. Segundo ciclo TOC.

Una vez planteadas las nuevas condiciones del proceso se pudo evidenciar, que el cuello de botella o restricción existente en la operación de esterilizado se reduce a niveles permisibles, al punto de no solo disminuir las horas empleadas, si no que permite también aumentar las unidades de producción en la presentación de $1 \mathrm{~kg}$. Sin embargo, como se observa en la figura (2) de medición de tiempos, la restricción 
del proceso ha migrado a las etapas de sellado y compactación.

En la tabla 4, se detallan los tiempos de operación requeridos para el proceso de sellado, considerando que dicha operación es realizada con un único equipo de una sola campana de sellado, la cual realiza la operación en ciclos.

Tabla 4.

Tiempo requerido por la selladora

\begin{tabular}{|c|c|c|c|c|c|}
\hline Formato & $\begin{array}{c}\text { Capacidad } \\
\text { (unidad) }\end{array}$ & $\begin{array}{c}\text { Unidad / } \\
\text { Campana }\end{array}$ & $\begin{array}{c}\text { Tiempo de } \\
\text { sellado } \\
\text { (min) }\end{array}$ & $\begin{array}{c}\text { Tiempo } \\
\text { requerido } \\
\text { (min) }\end{array}$ & $\begin{array}{c}\text { Tiempo } \\
\text { requerido } \\
\text { (horas) }\end{array}$ \\
\hline $1 \mathrm{KG}$ & 9520 & 4 & 0,5 & 1190 & 19,83 \\
\hline $3 \mathrm{KG}$ & 3360 & 3 & 0,5 & 56 & 9,33 \\
\hline $7 \mathrm{KG}$ & 1600 & 2 & 0,5 & 400 & 6,67 \\
\hline
\end{tabular}

De la misma forma, se muestran los tiempos relacionados con la operación de compactación, teniendo la consideración que dicho proceso es realizado de manera manual como se mencionó en el detalle de las operaciones (tabla 5).

Tabla 5.

Tiempo requerido por proceso de compactado manual

\begin{tabular}{|c|c|c|c|}
\hline Formato & Capacidad (Unidad) & Velocidad (Unidad/Min) & $\begin{array}{c}\text { Tiempo necesario } \\
\text { (Horas) }\end{array}$ \\
\hline $1 \mathrm{KG}$ & 9520 & $8 / \mathrm{min}$ & 19,83 \\
\hline $3 \mathrm{KG}$ & 3360 & $6 / \mathrm{min}$ & 9,33 \\
\hline $7 \mathrm{KG}$ & 1600 & $4 / \mathrm{min}$ & 6,67 \\
\hline
\end{tabular}

Como se observa en la tabla 8 , la operación de sellado realizado por una única maquina trabaja a la totalidad de su capacidad nominal.

En la tabla 4, para el caso de la compactación se debe de tener una consideración especial, por tratarse de una etapa del proceso, que es realizada enteramente por operarios (manual), estos pasan a ser la principal variable a considerar al momento de aumentar las cantidades a producir o reducir tiempos de operación, de modo que 
si se incrementa el volumen a producir y por ende se aumenta el número de operarios, se incrementará de manera directamente proporcional el costo y demás variables asociadas.

En este segundo ciclo se plantea como mejora requerida para el proceso la compra e instalación de un segundo equipo de compactación, junto con un equipo automatizado de compactación, con el fin de volver más eficientes estas etapas del proceso, reduciendo los recursos utilizados por los mismos, minimizando los tiempos empleados y el número de operarios y por ende los costos asociados (Garg, 2016).

A continuación, se representan los requerimientos de tiempo para los cambios planteados.
Tabla 6.

Tiempo requerido con dos selladoras

\begin{tabular}{|c|c|c|}
\hline Formato & $\begin{array}{c}\text { Tiempo } \\
\text { requerido } \\
\text { (horas) }\end{array}$ & $\begin{array}{c}\text { Tiempo } \\
\text { requerido } \\
\text { (horas) } x 2\end{array}$ \\
\hline $1 \mathrm{KG}$ & 29,78 & 14,89 \\
\hline $3 \mathrm{KG}$ & 14,03 & 7,02 \\
\hline $7 \mathrm{KG}$ & 10,04 & 5,02 \\
\hline
\end{tabular}

Como se observa en la tabla anterior, la capacidad de sellado virtualmente se duplica por tratarse de un equipo de similares características.

\section{Tabla 7.}

Velocidad de compactación (equipo)

\begin{tabular}{|c|c|}
\hline Formato & Velocidad (unidad/min) \\
\hline $1 \mathrm{~kg}$ & $10 / \mathrm{min}$ \\
\hline $3 \mathrm{~kg}$ & $8 / \mathrm{min}$ \\
\hline $7 \mathrm{~kg}$ & $6 / \mathrm{min}$ \\
\hline
\end{tabular}

Tabla 8.

Recursos requeridos compactación manual vs equipo

\begin{tabular}{|c|c|c|c|c|c|c|}
\hline \multicolumn{2}{|c|}{ Recursos utilizados } \\
\hline & \multicolumn{2}{|c|}{1600 fundas de $7 \mathrm{~kg}$} & \multicolumn{2}{c|}{3360 fundas de $3 \mathrm{~kg}$} & \multicolumn{2}{c|}{9520 fundas de $1 \mathrm{~kg}$} \\
\cline { 2 - 7 } & Manual & Compactadora & Manual & Compactadora & Manual & Compactadora \\
\hline $\begin{array}{c}\text { Tiempo } \\
\text { (horas) }\end{array}$ & 6,7 & 4,4 & 9,3 & 7,0 & 19,8 & 5,6 \\
\hline $\begin{array}{c}\text { Mano de } \\
\text { obra } \\
\text { (\#operarios) }\end{array}$ & 4 & 2 & 4 & 2 & 4 & 2 \\
\hline
\end{tabular}




\subsection{Tercer ciclo TOC}

Una vez realizadas las mejoras planteadas al proceso de conservas de pouch en el segundo ciclo o iteración, se realiza nuevamente el análisis de las condiciones del proceso.

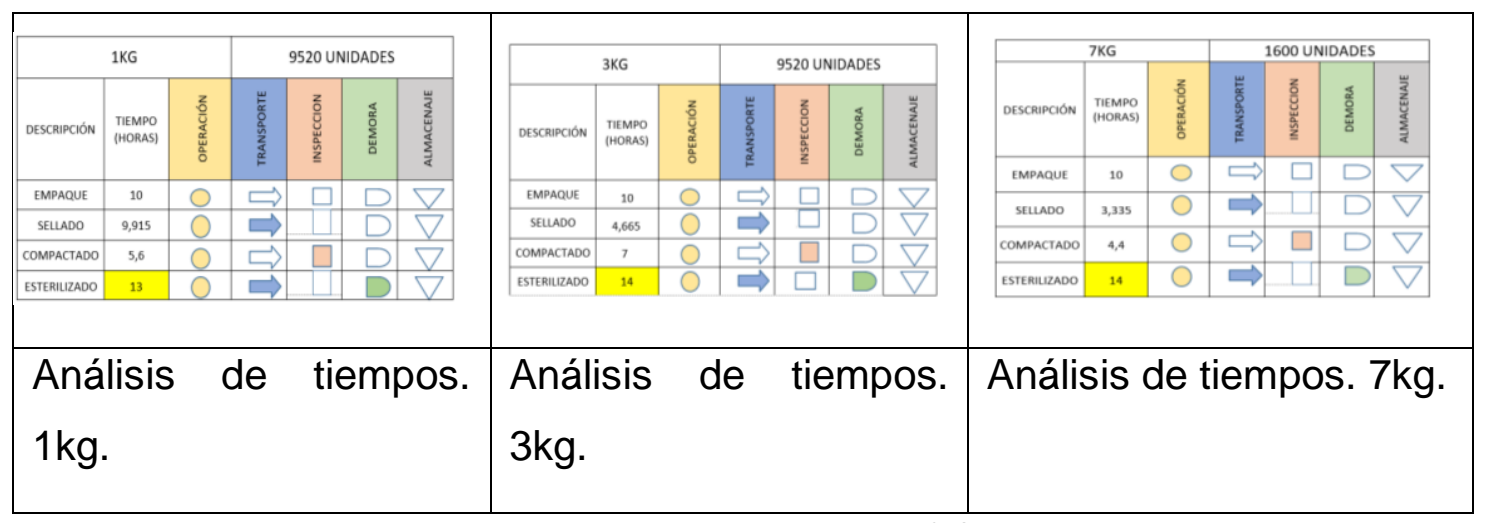

Figura 3. Tercer ciclo TOC

Como se puede apreciar en la figura 3, la restricción presente en la operación de sellado y compactado desaparece, es decir las capacidades del proceso han sido elevadas, con lo que el flujo de producción mantiene una dinámica uniforme en cada etapa.

\subsection{Impacto económico}

Una vez que el proyecto ya implantado es puesto en marcha, podemos realizar un análisis con información real de los procesos productivos (Figuera-Vinué, 2006). De este análisis se obtienen los siguientes resultados.

Tabla 9.

Resultados de producción 2017-2018

\begin{tabular}{|c|c|c|c|c|c|c|c|c|c|c|c|c|}
\hline Indicador & Jul-17 & Ago-17 & Sep-17 & Oct-17 & Nov-17 & Dic-17 & Ene-18 & Feb-18 & Mar-18 & Abr-18 & May-18 & $\begin{array}{c}\text { Jun-18 al } \\
20-J u n-18\end{array}$ \\
\hline $\begin{array}{c}\text { Unidades } \\
\text { Reales (kg) }\end{array}$ & 193799 & 152049 & 164474 & 105872 & 167942 & 244937 & 238214 & 214075 & 178430 & 252721 & 213175 & 240267 \\
\hline $\begin{array}{c}\text { Horas } \\
\text { laboradas de } \\
\text { Proceso }\end{array}$ & 388 & 360 & 383 & 312 & 385 & 404 & 247 & 233 & 195.3 & 235 & 221 & 274 \\
\hline $\begin{array}{c}\text { Días de } \\
\text { producción }\end{array}$ & 21 & 18 & 23 & 18 & 21 & 21 & 21 & 19 & 16 & 21 & 19 & 14 \\
\hline $\begin{array}{c}\text { Producción } \\
\text { real/día }\end{array}$ & 9229 & 8447 & 7151 & 5882 & 7997 & 11664 & 11344 & 11267 & 11152 & 12034 & 11220 & 17162 \\
\hline $\begin{array}{c}\text { Tasa } \\
\text { producción } \\
\mathrm{kg} / \text { hora }\end{array}$ & 500 & 423 & 429 & 339 & 436 & 606 & 963 & 918 & 914 & 1075 & 965 & 877 \\
\hline
\end{tabular}

Fuente: Indicadores de productividad Dpto. Producción. Fishcorp. S.A. 
Considerar que las unidades reales producidas en cada mes, se muestran en kilogramos, debido a que como ya se mencionó en otros apartados, para el producto pouch existen 3 presentaciones: $1 \mathrm{~kg}, 3 \mathrm{~kg}$ y $7 \mathrm{~kg}$, por lo que unificar los pesos facilita el análisis.

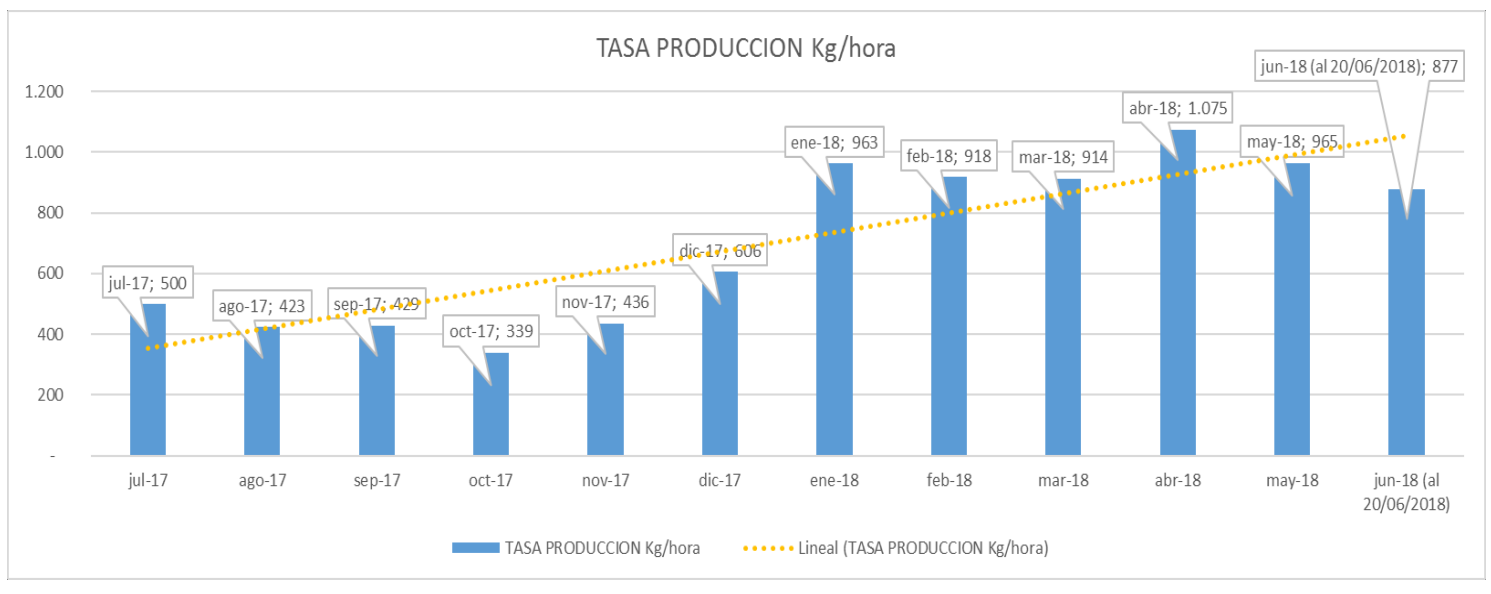

Figura 4. Evolución de la tasa de producción 2017-2018

La tasa de producción refleja la velocidad de producción de la línea de proceso. Como se puede apreciar en la figura 4, la tasa aumenta a más del doble a partir del segundo semestre, por tanto, podemos afirmar, que la empresa produce mayor cantidad en menos tiempo.

- Promedio de Julio a diciembre (2017): 456 kg/hora.

- Promedio de Enero a Junio (2018): $952 \mathrm{~kg} / \mathrm{hora}$.

Tabla 10.

Cálculo de desembolsos en mano de obra, período 2017-2018

\begin{tabular}{|c|c|c|c|c|c|}
\hline & Cantidades & Horas & $\begin{array}{c}\text { Valor } \\
\mathrm{H} / \mathrm{H}\end{array}$ & $\begin{array}{c}\# \text { de } \\
\text { Operadores }\end{array}$ & Desembolso \\
\hline 1er. Semestre & 1.029 .073 & 2231 & $\$ 1,53$ & 23 & $\$ 78.517,69$ \\
\hline $\begin{array}{c}\text { 2do. Semestre (sin } \\
\text { mejora) }\end{array}$ & 1.336 .882 & 2899 & $\$ 1,53$ & 23 & $\$ 102.003,36$ \\
\hline $\begin{array}{c}\text { 2do. Semestre } \\
\text { (con mejora) }\end{array}$ & 1.396 .949 & 1406 & $\$ 1,53$ & 23 & $\$ 49.470,45$ \\
\hline
\end{tabular}

En la tabla 10, se detalla el cálculo de los desembolsos correspondientes a mano de obra, asociado al excedente de horas que se generarían de no haber 
implementado las mejoras propuestas.

Como se aprecia, en el segundo semestre se realiza la diferenciación entre desembolso con mejora $(\$ 49.470,75)$ y $\sin$ mejora $(\$ 102.003,36)$. Siendo la diferencia entre estos el ahorro teórico en lo que se refiere a mano de obra $\$ 52.532,91$.

\section{Conclusiones}

La metodología de teoría de restricciones junto con la inclusión de control de tiempos aplicados a la propuesta de optimización ha sido de gran ayuda para la organización en su afán de mejorar continuamente sus procesos.

Se identificaron plenamente las restricciones del proceso usando como base técnica la metodología en cuestión (TOC), y como herramienta de apoyo estudio de tiempos. Se plantearon, y posteriormente se implementaron propuestas de mejora debidamente fundamentadas, que en términos generales muestran un avance evidente y comprobable.

Tomando como referencia el punto de partida existe una notable mejora en las capacidades productivas y los tiempos empleados, evolucionando de una tasa media de producción de $456 \mathrm{~kg} / \mathrm{h}$ a $952 \mathrm{~kg} / \mathrm{h}$, reduciendo así notablemente costos directos y maximizando los beneficios económicos de la empresa.

Se puede concluir indicando que metodologías y herramientas como las aplicadas, son completamente ajustables a cualquier tipo de organización, independientemente de su naturaleza o tamaño, siempre existe oportunidad de mejorar.

\section{Bibliografía}

Eliyahu, G. (2005). It's not luck. México: Ediciones Granica México s a.

Figuera Vinué, P. (2006). Optimización de productos y procesos industriales. Barcelona: Gestión 2000.

Fishcorp. (2017). Plan HACCP. Jaramijó-Ecuador: Fishcorp S.A.

Garg, H. (2016). Bi-criteria optimization for finding the optimal replacement interval for maintaining the performance of the process industries. In Handbook of Research on Modern Optimization Algorithms and Applications in Engineering and Economics (pp. 643-675). IGI Global. 
Goldratt, E. (1994). El síndrome del pajar. En E. Goldratt, El síndrome del pajar (págs. 5064). México.: Diaz de Santos.
Goldratt, E., \& Cox, J. (2008). La Meta. México: Ediciones Granica México s a. 